\title{
SURVEY AND POPULATION FLUCTUATION OF SOME RODENT SPECIES AT DIFFERENT LOCALITIES IN EL-IBRAHEMIA DISTRICT, SHARKIA GOVERNORATE
}

\author{
HEGAB, A.M.I. ${ }^{1}$, A.A.F, EI-BAHRAWY ${ }^{2}$, G.A. EL-KADY ${ }^{2}$ and \\ M.I.A. EL-BKHSHWANGI ${ }^{1}$ \\ 1- Plant Protection Research Institute, ARC, Dokki, Giza Egypt. \\ 2- Dept. of Plant Protection, Faculty of Agriculture, Suez Canal University, Egypt.
}

(Manuscript received 4 September 2013)

\begin{abstract}
The present investigation was carried out to survey the population fluctuation of some rodent species in different localities in El- Ibrahemia district, Sharkia Governorate.

Result revealed that the rodent species trapped from the three different habitats are classified as follows:

(A) The white bellied rat Rattus rattus frugivorus, (B) the grey bellied rat Rattus rattus alexandrinus, (C) the Norway rat Rattus norvegicus, (D) the house mouse Mus musculus and (E) the Egyptian spiny mouse Acomys cahrinus. The total number of rodent species in the houses during two successive years (20042005 and 2005 -2006) was 451 and 335 individuals, respectively. The white bellied Rattus rattus frugivorus was the most dominant species in this locality, while the total number of rodent species in the granaries during the same years was 115 and 120 individual, respectively. The white bellied rat Rattus rattus frugivorus was recorded the highest number of rodents. On the other hand, in poultry farm the total number of rodent species was 123 and 76 individuals during two successive years, respectively.

The highest values of total number of rats were recorded during May 2006 (98 and 73 individuals respectively), when the mean of temperature was $25.7^{\circ} \mathrm{C}$ during May 2005 and 2006, while the mean of relative humidity (RH) was 54.1 and $54.7 \%$, respectively.
\end{abstract}

\section{INTRODUCTION}

Rodents are cosmopolitan in their distribution and they constitue the largest group of mammals reaching nearly $40 \%$ of all mammals living at the present time (Bajomi, 1984). They characterized by rapid sexual maturation, short gestation period and increase frequent pregnancy in the year. The commensal rodents are of great importance, they play an important role in the spread of numerous diseases to man and domestic animals as well as causing considerable damage to agriculture crops specially sugarcane plantations, wheat, maize, rice, fruit trees and vegetables. They can be attack small animals, especially chickens, squabs of pigeon and suckling rabbits (Abdel- Azeem, 2008). 
Wild rats can attack all kinds of cereals and forage crops, granaries and stored food. On a world wide basis an annual loss of $3.55 \%$ of stored grains is due to the damage of these wild rats (Dykstra, 1966).

In Egypt, several authors studied the population density of different rodent species in some governorates Abdel-Gawad, 1974, Abdel-Gawad, et al. 1982, Mourad, et al. 1982, Asran, et al. 1985, El-Bahrawy, 1986, Abdel-Karim, 1991, Asran, et al. 1991, Asran, 1994, Hegab, 2004 and Hegab et al. 2006. The aim of the present work is an attempt to throwlight on the problems of rodents in sewi- area at Sharkia governorate, Ibrahemia district during two successive seasons of 2005/2006 in the three different localities, i.e. houses, granaries and poultry farm).

The aim of study as following:

1- Survey and population fluctuation of the rodent species during two successive seasons $2004-2005$ and 2005 / 2006 at the three different localities in Ibrahemia, district, Sharkia governorate.

2- Effect of temperature and relative humidity on the total numbers of rodent species during two successive years.

\section{MATERIALS AND METHODS}

\section{1- Survey:}

The present study was carried out in three different localities, granaries, houses and poultry farm at El-Ibrahemia district, Sharkia governorate during two successive season $2004 / 2005$ and 2005 /2006. The seasonal fluctuation of rodent was carried out for two years from November 2004 up to the end of October 2006. The first locality was cereal granaries (about 5 granaries), the second site was urban area including many houses (about 100 houses). The third site was a poultry farm about $300 \mathrm{~m}^{2}$ consists of two floors building and feed storage.

Rodents were trapped alive from the three localities, forty clean wire-box traps with spring doors were used at each locality for two times per month (at the beginning and the half of month), traps were cleaned by water and liquid soap before each use. The traps were supplied with fresh bait materials, namely: tomato slices. In each investigated site, the traps were baits with fresh bait and distributed two times in different positions, in the granaries the traps were setup beside the wall at distances of $10 \mathrm{~m}$ from another trap, in the houses the traps were setup in the infested places, in the poultry farm the traps were setup in the food storage granary and inside the farm beside the wall. The traps were left from 6p.m. in above mentioned positions and collected in the morning at 7 a.m. Captured traps were 
separately enclosed in white cloth bags fastened with a string and transferred carefully in the same day to the laboratory for examination, El-Bahrawy et al. (2008).

The trapped rats were individually inserted in to a killing jar provided with a piece cotton wool moistened with chlorophorm for anesthetizing rats.

\section{The estimated data from the trapped rats were:}

a- Survey, indentification and classification into species according to the Osbron and Helmy (1980).

b- Population density of the rodent species.

\section{2- Effect of temperature and relative humidity on the total numbers of} rodent species during two successive years.

The collected rodents were estimated for each season and studied locality and analyzed by using factorial analysis method. The influence of temperature and relative humidity on the activity of rodent species trapped during two successive years of study was determined. The data of temperature and relative humidity were taking from the central Lab. For Agric. Climate, Agric, Res. Center, Giza, Egypt.

\section{RESULTS AND DISCUSSION}

\section{Survey.}

Rodent species in three different sites in El-Ibrahemia district, Sharkia Governorate were surveyed, the population fluctuation was studied during two successive years 2004 /2005 and 2005 /2006.

Rodent species were the white bellied rat Rattus rattus frugivorus, the grey bellied rat Rattus rattus alexandarinus, the Norwayrat Rattus norvegicus, the house mouse Mus musculus and the spiny mouse Acomys cahrinus.

The rodent species were identified according to the full description of rodent species of Egypt adopted by Osbron and Helmy (1980).

\section{Population fluctuation of rodent species at three different sites.}

Data presented in Tables ( $1 \& 2$ ) indicated that the relative abundance of different rodent species at the three different localities in El-Ibrahemia district, Sharkia governorate during years of 2004 / 2005 and 2005 /2006. The average of rat index was 0.24 and 0.18 during the $1^{\text {st }}$ year and the $2^{\text {nd }}$ year, respectively. The highest mean of rat index were 0.47 and .35 during the $1^{\text {st }}$ and $2^{\text {nd }}$ years, respectively in the houses followed by the poultry farm $(0.13)$, the granaries $(0.12)$ during the $1^{\text {st }}$ year, and while during the $2^{\text {nd }}$ years the granaries and poultry farm were recorded 0.13 and 0.18 , respectively. 
The highest value of rat index of all sites were obtained 0.41 and 0.30 during May 2005 and 2006, respectively, while the lowest value of rat index was 0.12 recorded during November 2004 and (0.08) during March 2006.

It appears also that the highest rat index in the houses were observed during August 2005 and 2006 (0.86 and 0.70, respectively), while the lowest rat index was 0.16 and 0.11 during March 2005 and February 2006. The highest rat index in the granaries were 0.25 during May 2005 and 0.20 during January 2006, the lowest rat index in the granaries was 0.01 and zero during June 2005 and March 2006, respectively. While the highest rat index in the poultry farm was 0.25 during the three months May 2005 \& 2006 and June 2005, the lowest rat index in the poultry farm was 0.04 during February 2005 and the period from August up to October 2006 (zero).

\subsubsection{In houses.}

Data in Tables ( $3 \& 4)$ indicated that the total number of rodent species in the houses during two successive years 2004 / 2005 and 2005/2006 were 451 and 335 individuals, respectively. The white bellied rat, Rattus rattus frugivorus was the most dominant species in this site, followed by the grey bellied rat, $R$. rattus. frugivours, the house mouse, rat, Mus musculus and the Egyptian spiny mous, Acomys cahrinus.

The maximum number of total rodent species was 69 and 56 individuals during August 2005 and 2006, respectively, while the minimum number was 13 and 9 during March 2005 and February 2006, respectively.

\subsubsection{In Granaries.}

Data president in Tables $(5 \& 6)$ showed that the total number of rodent species in the granaries during two successive years was 115 and 120 individuals, respectively. The white bellied rat Rattus rattus frugivorus recorded the highest number of rodent, followed by $R$. norvegicus and $R$. rattus. alexandrinus. The highest population of total rodent species was recorded during May 2005 (20 individuals), November 2005 and January 2006 (16 individuals), while the lowest population was obtained during June 2005 and March 2006 (1 and zero, respectively).

\subsubsection{In poultry farm.}

Data in Tables $(7 \& 8)$ showed that the total number of rodent species in the poultry farm during two successive years was 123 and 76 individuals during the $1^{\text {st }}$ and $2^{\text {nd }}$ years, respectively. The white bellied rat $R . r$. frugivorus was the most dominant species in the poultry farm during the two years, followed by $R$. rattus. alexandrinus and $R$. norvegicus. The maximum number of total rodent species was recorded during May 2005, 2006 and June 2005 (20 individuals), while the minimum number was noticed during February 2005 (3 individuals) and no observed during of three months (August, September and October 2006). Also, it is that the rodent 
species disappeared during the period from August up to October 2006 in the poultry farm. Most of poultry farms are destroyed by virus fowel influenza (H5N2) during this time.

These findings agree with those obtained by Gaaboub et al. (1978), Asran et al. (1985), El-Bahrawy (1986), Soliman et al. (2001), Hegab (2004) and Hegab et al. (2006).

Gaaboub et al. (1978) made a survey of rodent in Alexandria governorate, Egypt and found six species of rodents namely: $R$. $r$. frugivorus, $R$. norvegicus, $R . r$. alexandrinus, A. niloticus, R. r. rattus and Mus musculus.

Asran et al. (1985) in Fayoum governorate, Egypt studied rodent population density of $A$. niloticus in different location and showed that the rodent population density differed from location to location, from district to another and from month to month. El-Bahrawy (1986) found that the relative abundance of rodent species in Ismailia governorate may be arranged in the following descending order: $R$. $r$. frugivorus, $R$. norvegicus, $R$. $r$. alexandrinus and Mus musculus, respectively. It concluded that $R$. r. frugivorus was the most dominant species and Mus musculus was the least dominant. Soliman et al. (2001) in his work in 3 villages, namely kafr Ayoub Soliman, Kafr Ibrahim El-Aidi, and El-Saadat, Sharkia governorate, Egypt. A total of 519 rats were collected from the 3 study sites: $46.6 \% R$. rattus, and $53.4 \% R$. norvegicus.

Hegab (2004) in his study in three different localities during winter, 2003 - 2004 at Sharkia governorate showed that the highest population density were in houses (198), in granaries and citrus farm (54).

Hegab et al. (2006) in his work in three different sites at Sharkia governorate (i.e. houses, citrus farm and granaries). Results revealed that the houses gave the highest values of numbers for rodents (192) followed by citrus farm (51) and the granaries (42).

Mmetwaly, et al. (2009) found that seven rodent species belonging to 6 genera and 2 families at Behera governorate. Firstly, concerning family Muridae, four genera were found namely Rattus, Arvicanthis, Acomys and Mus, Rattus rattus (Linn.), in addition to Rattus norvegicus (Berk)., as well as Mus musculus (Linn.), were recorded in the buildings, while Meriones shawi isis (Thomas) and Gerbillus gerbillus (Olivier) were found in field crops.

\section{Effect of temperature and relative humidity on the total numbers of rodent species during two successive years.}

It appears from the results given in Tables $(9 \& 10)$ and Figs $(1 \& 2)$ that the relation between monthly fluctuation abundance of the rodent species and 
temperature \& relative humidity $(\mathrm{RH})$ in Sharkia governorate during two successive years $2004 / 2005$ and $2005 / 2006$. The results revealed that the highest total number of rats were recorded during May 2005 and 2006 (98 and 73, respectively), when the mean of temperature was $25.7^{\circ} \mathrm{C}$ during May 2005 and 2006, while the mean of relative humidity was 54.0 and $54.7 \%$, respectively. The lowest percentage of rats were 12.1 and 7.5\% obtained during November 2004 and March 2006, respectively, when the mean of temperature was $22^{\circ} \mathrm{C}$ during November 2004 and $20.1^{\circ} \mathrm{C}$ during March 2006. Also, the mean of relative humidity was 65 and $58.6 \%$ during November 2004 and March 2006, respectively. These results agree with those obtained by AbdelGawad (1974 and 1979) found that, the highest density was observed in Summer and the lowest one was recorded in winter. Omar (1980) in his survey of domestic and commensal rodents in the $10^{\text {th }}$ of Ramadan city found that the population of the rodents could be arranged according to the different seasons in a descending order in 1979 as follow: spring, summer, autumn and winter. Embarak (1997) reported that the fluctuation of the number of rodent in cultivated area was studied for two successive years and showed that the highest density was recorded in summer and least density in winter during the two years, while in newly reclaimed area the highest population density of rodents was recorded in spring and the least occurred in winter during the studied year. 
Table 1. Population fluctuation of the different rodent species in El-Ibrahemia district, Sharkia Governorate during year of 2004 / 2005.

\begin{tabular}{|c|c|c|c|c|c|c|c|c|c|c|c|c|c|c|c|c|c|}
\hline \multirow[b]{2}{*}{ Months } & \multicolumn{5}{|c|}{ Houses } & \multicolumn{5}{|c|}{ Granaries } & \multicolumn{5}{|c|}{ Poultry farm } & \multicolumn{2}{|c|}{ total } \\
\hline & M. & F. & $\mathrm{T}$. & $\begin{array}{c}\text { No. of } \\
\text { positive traps }\end{array}$ & Rat index. & M. & F. & T. & $\begin{array}{c}\text { No. of } \\
\text { positive traps }\end{array}$ & Rat index & M. & F. & $\mathrm{T}$. & $\begin{array}{c}\text { No. of } \\
\text { positive traps }\end{array}$ & Rat index & $\begin{array}{c}\text { No. of } \\
\text { rats }\end{array}$ & Rat index \\
\hline Nov. 04 & 7 & 7 & 14 & 14 & 0.18 & 8 & 2 & 10 & 10 & 0.13 & 4 & 1 & 5 & 5 & 0.06 & 29 & 0.12 \\
\hline Dec. & 7 & 9 & 16 & 15 & 0.20 & 5 & 5 & 10 & 10 & 0.13 & 4 & 6 & 10 & 10 & 0.13 & 36 & 0.15 \\
\hline Jan. 05 & 14 & 11 & 25 & 24 & 0.31 & 1 & 4 & 5 & 5 & 0.06 & 7 & 3 & 10 & 10 & 0.13 & 40 & 0.17 \\
\hline Feb. & 13 & 16 & 29 & 28 & 0.36 & 4 & 2 & 6 & 6 & 0.08 & 1 & 2 & 3 & 3 & 0.04 & 38 & 0.16 \\
\hline Mar. & 6 & 7 & 13 & 12 & 0.16 & 5 & 6 & 11 & 11 & 0.14 & 5 & 3 & 8 & 8 & 0.10 & 32 & 0.13 \\
\hline Apr. & 21 & 31 & 52 & 49 & 0.65 & 4 & 9 & 13 & 13 & 0.16 & 7 & 7 & 14 & 14 & 0.18 & 79 & 0.33 \\
\hline May & 24 & 34 & 58 & 54 & 0.73 & 14 & 6 & 20 & 20 & 0.25 & 7 & 13 & 20 & 20 & 0.25 & 98 & 0.41 \\
\hline June & 20 & 29 & 49 & 45 & 0.61 & 0 & 1 & 1 & 1 & 0.01 & 10 & 10 & 20 & 20 & 0.25 & 70 & 0.29 \\
\hline July & 19 & 20 & 39 & 39 & 0.49 & 5 & 5 & 10 & 10 & 0.13 & 2 & 3 & 5 & 5 & 0.06 & 54 & 0.23 \\
\hline Aug & 31 & 38 & 69 & 65 & 0.86 & 5 & 5 & 10 & 10 & 0.13 & 4 & 8 & 12 & 12 & 0.15 & 91 & 0.38 \\
\hline Sep. & 25 & 27 & 52 & 46 & 0.65 & 6 & 10 & 16 & 16 & 0.20 & 5 & 5 & 10 & 10 & 0.13 & 78 & 0.33 \\
\hline Oct. & 18 & 17 & 35 & 32 & 0.44 & 1 & 2 & 3 & 3 & 0.04 & 3 & 3 & 6 & 6 & 0.08 & 44 & 0.18 \\
\hline Total & 205 & 246 & 451 & & & 58 & 57 & 115 & & & 59 & 64 & 123 & & & 689 & \\
\hline Mean & & & & & 0.47 & & & & & 0.12 & & & & & 0.13 & & 0.24 \\
\hline
\end{tabular}

M. Males, F.: Females, T. : Total 
Table 2. Population fluctuation of the different rodent species in El-Ibrahemia district, Sharkia Governorate during year of 2005 /2006.

\begin{tabular}{|c|c|c|c|c|c|c|c|c|c|c|c|c|c|c|c|c|c|}
\hline \multirow[b]{2}{*}{ Months } & \multicolumn{5}{|c|}{ Houses } & \multicolumn{5}{|c|}{ Granaries } & \multicolumn{5}{|c|}{ Poultry farm } & \multicolumn{2}{|c|}{ total } \\
\hline & M. & F. & $\mathrm{T}$ & $\begin{array}{c}\text { No. of } \\
\text { positive } \\
\text { traps }\end{array}$ & Rat index. & M. & F. & $\mathrm{T}$. & $\begin{array}{c}\text { No. of } \\
\text { positive } \\
\text { traps }\end{array}$ & $\begin{array}{l}\text { Rat } \\
\text { index }\end{array}$ & M. & $\mathrm{F}$. & $\mathrm{T}$. & $\begin{array}{c}\text { No. of } \\
\text { positive } \\
\text { traps }\end{array}$ & $\begin{array}{c}\text { Rat } \\
\text { index }\end{array}$ & $\begin{array}{l}\text { No. } \\
\text { of } \\
\text { rats }\end{array}$ & $\begin{array}{l}\text { Rat } \\
\text { index }\end{array}$ \\
\hline Nov. 05 & 7 & 10 & 17 & 15 & 0.21 & 11 & 5 & 16 & 16 & 0.20 & 0 & 5 & 5 & 5 & 0.06 & 38 & 0.16 \\
\hline Dec. & 10 & 10 & 20 & 19 & 0.25 & 5 & 9 & 14 & 14 & 0.18 & 7 & 4 & 11 & 11 & 0.14 & 45 & 0.19 \\
\hline Jan. 06 & 5 & 10 & 15 & 15 & 0.19 & 7 & 9 & 16 & 16 & 0.20 & 5 & 2 & 7 & 7 & 0.09 & 38 & 0.16 \\
\hline Feb. & 6 & 3 & 9 & 9 & 0.11 & 9 & 5 & 14 & 14 & 0.18 & 5 & 1 & 6 & 6 & 0.08 & 29 & 0.12 \\
\hline Mar. & 7 & 3 & 10 & 10 & 0.13 & 0 & 0 & 0 & 0 & 0.00 & 3 & 5 & 8 & 8 & 0.10 & 18 & 0.08 \\
\hline Apr. & 24 & 17 & 41 & 39 & 0.51 & 3 & 4 & 7 & 7 & 0.09 & 3 & 7 & 10 & 10 & 0.13 & 58 & 0.24 \\
\hline May & 16 & 25 & 41 & 41 & 0.51 & 10 & 2 & 12 & 12 & 0.15 & 7 & 13 & 20 & 20 & 0.25 & 73 & 0.30 \\
\hline June & 23 & 14 & 37 & 36 & 0.46 & 4 & 1 & 5 & 5 & 0.06 & 3 & 5 & 8 & 8 & 0.10 & 50 & 0.21 \\
\hline July & 16 & 16 & 32 & 30 & 0.40 & 7 & 0 & 7 & 7 & 0.09 & 1 & 0 & 1 & 1 & 0.01 & 40 & 0.17 \\
\hline Aug & 28 & 28 & 56 & 55 & 0.70 & 3 & 4 & 7 & 7 & 0.09 & 0 & 0 & 0 & 0 & 0.00 & 63 & 0.26 \\
\hline Sep. & 14 & 16 & 30 & 30 & 0.38 & 7 & 1 & 8 & 8 & 0.10 & 0 & 0 & 0 & 0 & 0.00 & 38 & 0.16 \\
\hline Oct. & 9 & 18 & 27 & 26 & 0.34 & 9 & 5 & 14 & 14 & 0.18 & 0 & 0 & 0 & 0 & 0.00 & 41 & 0.17 \\
\hline Total & 165 & 170 & 335 & & & 75 & 45 & 120 & & & 34 & 42 & 76 & & & 531 & \\
\hline Mean & & & & & 0.35 & & & & & 0.13 & & & & & 0.18 & & 0.18 \\
\hline
\end{tabular}

M. Males, F.: Females, T. : Total 
Table 3. Population fluctuation of the different rodent species in the houses during year of 2004 / 2005.

\begin{tabular}{|c|c|c|c|c|c|c|c|c|c|c|c|c|c|}
\hline \multirow{2}{*}{ Months } & \multicolumn{3}{|c|}{ R. r. frugivorus } & \multicolumn{3}{|c|}{$\begin{array}{c}\text { R. r. } \\
\text { alexandrinus }\end{array}$} & \multicolumn{3}{|c|}{ Acomys cahrinus } & \multicolumn{3}{|c|}{ Mus musculus } & \multirow{2}{*}{$\begin{array}{l}\text { Grand } \\
\text { total }\end{array}$} \\
\hline & M. & $\mathrm{F}$. & $\mathrm{T}$. & M. & $\mathrm{F}$. & $\mathrm{T}$. & M. & $\mathrm{F}$. & $\mathrm{T}$. & M. & F. & $\mathrm{T}$. & \\
\hline Nov.04 & 5 & 7 & 12 & 2 & 0 & 2 & 0 & 0 & 0 & 0 & 0 & 0 & 14 \\
\hline Dec. & 5 & 6 & 11 & 1 & 1 & 2 & 0 & 0 & 0 & 1 & 2 & 3 & 16 \\
\hline Jan.05 & 10 & 7 & 17 & 1 & 1 & 2 & 0 & 1 & 1 & 3 & 2 & 5 & 25 \\
\hline Feb. & 10 & 13 & 23 & 1 & 2 & 3 & 1 & 1 & 2 & 1 & 0 & 1 & 29 \\
\hline Mar. & 4 & 5 & 9 & 2 & 1 & 3 & 0 & 1 & 1 & 0 & 0 & 0 & 13 \\
\hline Apr. & 20 & 27 & 47 & 0 & 4 & 4 & 0 & 0 & 0 & 1 & 0 & 1 & 52 \\
\hline May & 21 & 29 & 50 & 2 & 5 & 7 & 0 & 0 & 0 & 1 & 0 & 1 & 58 \\
\hline June & 18 & 25 & 43 & 2 & 4 & 6 & 0 & 0 & 0 & 0 & 0 & 0 & 49 \\
\hline July & 18 & 16 & 34 & 1 & 4 & 5 & 0 & 0 & 0 & 0 & 0 & 0 & 39 \\
\hline Aug & 27 & 26 & 53 & 4 & 11 & 15 & 0 & 1 & 1 & 0 & 0 & 0 & 69 \\
\hline Sep. & 21 & 23 & 44 & 4 & 4 & 8 & 0 & 0 & 0 & 0 & 0 & 0 & 52 \\
\hline Oct. & 16 & 14 & 30 & 2 & 3 & 5 & 0 & 0 & 0 & 0 & 0 & 0 & 35 \\
\hline Total & 175 & 198 & 373 & 22 & 40 & 62 & 1 & 4 & 5 & 7 & 4 & 11 & 451 \\
\hline
\end{tabular}

Table 4. Population fluctuation of the different rodent species in the houses during year of 2005 / 2006.

\begin{tabular}{|c|c|c|c|c|c|c|c|c|c|c|c|c|c|}
\hline \multirow{2}{*}{ Months } & \multicolumn{3}{|c|}{ R. r. frugivorus } & \multicolumn{3}{|c|}{$\begin{array}{c}\text { R. r. } \\
\text { alexandrinus }\end{array}$} & \multicolumn{3}{|c|}{ Acomys cahrinus } & \multicolumn{3}{|c|}{ Mus musculus } & \multirow{2}{*}{$\begin{array}{c}\text { Grand } \\
\text { total }\end{array}$} \\
\hline & M. & F. & $\mathrm{T}$. & M. & F. & T. & M. & F. & T. & M. & F. & T. & \\
\hline Nov.05 & 6 & 10 & 16 & 1 & 0 & 1 & 0 & 0 & 0 & 0 & 0 & 0 & 17 \\
\hline Dec. & 9 & 10 & 19 & 0 & 0 & 0 & 1 & 0 & 1 & 0 & 0 & 0 & 20 \\
\hline Jan.06 & 5 & 8 & 13 & 0 & 2 & 2 & 0 & 0 & 0 & 0 & 0 & 0 & 15 \\
\hline Feb. & 5 & 3 & 8 & 1 & 0 & 1 & 0 & 0 & 0 & 0 & 0 & 0 & 9 \\
\hline Mar. & 7 & 2 & 9 & 0 & 0 & 0 & 0 & 1 & 1 & 0 & 0 & 0 & 10 \\
\hline Apr. & 22 & 15 & 37 & 2 & 2 & 4 & 0 & 0 & 0 & 0 & 0 & 0 & 41 \\
\hline May & 15 & 23 & 38 & 1 & 2 & 3 & 0 & 0 & 0 & 0 & 0 & 0 & 41 \\
\hline June & 20 & 13 & 33 & 3 & 1 & 4 & 0 & 0 & 0 & 0 & 0 & 0 & 37 \\
\hline July & 15 & 15 & 30 & 1 & 1 & 2 & 0 & 0 & 0 & 0 & 0 & 0 & 32 \\
\hline Aug & 28 & 24 & 52 & 0 & 4 & 4 & 0 & 0 & 0 & 0 & 0 & 0 & 56 \\
\hline Sep. & 13 & 15 & 28 & 0 & 1 & 1 & 0 & 0 & 0 & 1 & 0 & 1 & 30 \\
\hline Oct. & 7 & 14 & 21 & 1 & 2 & 3 & 1 & 2 & 3 & 0 & 0 & 0 & 27 \\
\hline Total & 152 & 152 & 304 & 10 & 15 & 25 & 2 & 3 & 5 & 1 & 0 & 1 & 335 \\
\hline
\end{tabular}

M. Males, F.: Females, T.: Total 
Table 5. Population fluctuation of the different rodent species in the granaries during year of 2004 / 2005.

\begin{tabular}{|c|c|c|c|c|c|c|c|c|c|c|}
\hline & \multicolumn{3}{|c|}{ R. r. frugivorus } & \multicolumn{3}{c|}{ R. r. alexandrinus } & \multicolumn{3}{c|}{ R. norvegicus } & \multirow{2}{*}{ Grand total } \\
\cline { 2 - 12 } Months & M. & F. & T. & M. & F. & T. & M. & F. & T. & \\
\hline Nov.04 & 5 & 2 & 7 & 0 & 0 & 0 & 3 & 0 & 3 & 10 \\
\hline Dec. & 3 & 2 & 5 & 0 & 0 & 0 & 2 & 3 & 5 & 10 \\
\hline Jan.05 & 1 & 2 & 3 & 0 & 0 & 0 & 0 & 2 & 2 & 5 \\
\hline Feb. & 2 & 1 & 3 & 0 & 0 & 0 & 2 & 1 & 3 & 6 \\
\hline Mar. & 0 & 3 & 3 & 0 & 0 & 0 & 5 & 3 & 8 & 11 \\
\hline Apr. & 3 & 9 & 12 & 1 & 0 & 1 & 0 & 0 & 0 & 13 \\
\hline May & 12 & 5 & 17 & 2 & 1 & 3 & 0 & 0 & 0 & 20 \\
\hline June & 0 & 1 & 1 & 0 & 0 & 0 & 0 & 0 & 0 & 1 \\
\hline July & 0 & 3 & 3 & 0 & 0 & 0 & 5 & 2 & 7 & 10 \\
\hline Aug & 3 & 5 & 8 & 2 & 0 & 2 & 0 & 0 & 0 & 10 \\
\hline Sep. & 5 & 8 & 13 & 0 & 0 & 0 & 1 & 2 & 3 & 16 \\
\hline Oct. & 1 & 2 & 3 & 0 & 0 & 0 & 0 & 0 & 0 & 3 \\
\hline Total & 35 & 43 & 78 & 5 & 1 & 6 & 18 & 13 & 31 & 115 \\
\hline
\end{tabular}

Table 6. Population fluctuation of the different rodent species in the granaries during year of 2005 / 2006.

\begin{tabular}{|c|c|c|c|c|c|c|c|c|c|c|}
\hline \multirow{2}{*}{$\begin{array}{c}\text { Month } \\
\text { S }\end{array}$} & \multicolumn{2}{|c|}{ R. r. frugivorus } & \multicolumn{2}{c|}{ R. r. alexandrinus } & \multicolumn{3}{|c|}{ R. norvegicus } & \multirow{2}{*}{ Grand } \\
\cline { 2 - 12 } & M. & F. & T. & M. & F. & T. & M. & F. & T. & total \\
\hline 5 & 8 & 5 & 13 & 0 & 0 & 0 & 3 & 0 & 3 & 16 \\
\hline Dec. & 1 & 4 & 5 & 2 & 0 & 2 & 2 & 5 & 7 & 14 \\
\hline Jan.06 & 3 & 0 & 3 & 2 & 1 & 3 & 2 & 8 & 10 & 16 \\
\hline Feb. & 4 & 1 & 5 & 0 & 0 & 0 & 5 & 4 & 9 & 14 \\
\hline Mar. & 0 & 0 & 0 & 0 & 0 & 0 & 0 & 0 & 0 & 0 \\
\hline Apr. & 2 & 0 & 2 & 0 & 0 & 0 & 1 & 4 & 5 & 7 \\
\hline May & 6 & 2 & 8 & 1 & 0 & 1 & 3 & 0 & 3 & 12 \\
\hline June & 0 & 0 & 0 & 0 & 0 & 0 & 4 & 1 & 5 & 5 \\
\hline July & 3 & 0 & 3 & 0 & 0 & 0 & 4 & 0 & 4 & 7 \\
\hline Aug & 3 & 2 & 5 & 0 & 2 & 2 & 0 & 0 & 0 & 7 \\
\hline Sep. & 3 & 0 & 3 & 0 & 0 & 0 & 4 & 1 & 5 & 8 \\
\hline Oct. & 8 & 4 & 12 & 0 & 0 & 0 & 1 & 1 & 2 & 14 \\
\hline Total & 41 & 18 & 59 & 5 & 3 & 8 & 29 & 24 & 53 & 120 \\
\hline
\end{tabular}

M. Males, F.: Females, T.: Total 
Table 7. Population fluctuation of the different rodent species in the poultry farm during year of 2004 / 2005.

\begin{tabular}{|c|c|c|c|c|c|c|c|c|c|c|}
\hline & \multicolumn{3}{|c|}{ R. r. frugivorus } & \multicolumn{3}{c|}{ R. r. alexandrinus } & \multicolumn{3}{c|}{ R. norvegicus } & \multirow{2}{*}{ Grand total } \\
\cline { 2 - 12 } Months & M. & F. & T. & M. & F. & T. & M. & F. & T. & \\
\hline Nov.04 & 2 & 0 & 2 & 2 & 1 & 3 & 0 & 0 & 0 & 5 \\
\hline Dec. & 0 & 0 & 0 & 1 & 4 & 5 & 3 & 2 & 5 & 10 \\
\hline Jan.05 & 2 & 3 & 5 & 2 & 0 & 2 & 3 & 0 & 3 & 10 \\
\hline Feb. & 1 & 2 & 3 & 0 & 0 & 0 & 0 & 0 & 0 & 3 \\
\hline Mar. & 3 & 3 & 6 & 0 & 0 & 0 & 2 & 0 & 2 & 8 \\
\hline Apr. & 3 & 0 & 3 & 1 & 2 & 3 & 3 & 5 & 8 & 14 \\
\hline May & 2 & 6 & 8 & 2 & 5 & 7 & 3 & 2 & 5 & 20 \\
\hline June & 0 & 5 & 5 & 3 & 2 & 5 & 7 & 3 & 10 & 20 \\
\hline July & 0 & 0 & 0 & 0 & 2 & 2 & 2 & 1 & 3 & 5 \\
\hline Aug & 2 & 1 & 3 & 1 & 7 & 8 & 1 & 0 & 1 & 12 \\
\hline Sep. & 5 & 2 & 7 & 0 & 3 & 3 & 0 & 0 & 0 & 10 \\
\hline Oct. & 1 & 0 & 1 & 2 & 3 & 5 & 0 & 0 & 0 & 6 \\
\hline Total & 21 & 22 & 43 & 14 & 29 & 43 & 24 & 13 & 37 & 123 \\
\hline
\end{tabular}

Table 8. Population fluctuation of the different rodent species in the poultry farm during year of 2005 / 2006.

\begin{tabular}{|c|c|c|c|c|c|c|c|c|c|c|}
\hline & \multicolumn{3}{|c|}{ R. r. frugivorus } & \multicolumn{3}{|c|}{ R. r. alexandrinus } & \multicolumn{3}{c|}{ R. norvegicus } & \multirow{2}{*}{ Grand total } \\
\cline { 2 - 12 } Months & M. & F. & T. & M. & F. & T. & M. & F. & T. & \\
\hline Nov.05 & 0 & 3 & 3 & 0 & 2 & 2 & 0 & 0 & 0 & 5 \\
\hline Dec. & 4 & 1 & 5 & 3 & 3 & 6 & 0 & 0 & 0 & 11 \\
\hline Jan.06 & 5 & 0 & 5 & 0 & 2 & 2 & 0 & 0 & 0 & 7 \\
\hline Feb. & 5 & 1 & 6 & 0 & 0 & 0 & 0 & 0 & 0 & 6 \\
\hline Mar. & 3 & 5 & 8 & 0 & 0 & 0 & 0 & 0 & 0 & 8 \\
\hline Apr. & 3 & 2 & 5 & 0 & 3 & 3 & 0 & 2 & 2 & 10 \\
\hline May & 3 & 7 & 10 & 1 & 6 & 7 & 3 & 0 & 3 & 20 \\
\hline June & 0 & 2 & 2 & 1 & 0 & 1 & 2 & 3 & 5 & 8 \\
\hline July & 1 & 0 & 1 & 0 & 0 & 0 & 0 & 0 & 0 & 1 \\
\hline Aug & 0 & 0 & 0 & 0 & 0 & 0 & 0 & 0 & 0 & 0 \\
\hline Sep. & 0 & 0 & 0 & 0 & 0 & 0 & 0 & 0 & 0 & 0 \\
\hline Oct. & 0 & 0 & 0 & 0 & 0 & 0 & 0 & 0 & 0 & 0 \\
\hline Total & 24 & 21 & 45 & 5 & 16 & 21 & 5 & 5 & 10 & 76 \\
\hline
\end{tabular}

M. Males, F.: Females, T.: Total 
Table 9. Relation between averages of monthly fluctuation abundance and temperature \& relative humidity $(\mathrm{RH})$ of some rodent species in Sharkia Governorate during (Nov. 2004- Oct. 2005).

\begin{tabular}{|c|c|c|c|c|c|c|c|c|}
\hline Months & $\begin{array}{c}\text { Min } \\
\text { temp }\end{array}$ & $\begin{array}{c}\text { Max } \\
\text { temp }\end{array}$ & $\begin{array}{c}\text { Mean } \\
\text { temp }\end{array}$ & $\begin{array}{c}\text { Min } \\
\mathrm{RH} \%\end{array}$ & $\begin{array}{c}\text { Max } \\
\mathrm{RH} \%\end{array}$ & $\begin{array}{c}\text { Mean } \\
\mathrm{RH} \%\end{array}$ & $\begin{array}{c}\text { Total No. } \\
\text { of rats }\end{array}$ & $\%$ \\
\hline Nov.04 & 17.2 & 26.7 & 22.0 & 45.0 & 85.0 & 65.0 & 29 & 12.1 \\
\hline Dec. & 13.2 & 22.9 & 18.1 & 39.5 & 82.7 & 61.1 & 36 & 15.0 \\
\hline Jan.05 & 10.5 & 19.4 & 14.9 & 41.3 & 80.5 & 60.9 & 40 & 16.7 \\
\hline Feb. & 11.2 & 20.9 & 16.0 & 38.8 & 83.8 & 61.3 & 38 & 15.8 \\
\hline Mar. & 13.3 & 24.0 & 18.7 & 37.6 & 82.7 & 60.2 & 32 & 13.3 \\
\hline Apr. & 15.2 & 28.5 & 21.9 & 29.3 & 83.3 & 56.3 & 79 & 32.9 \\
\hline May & 18.8 & 32.6 & 25.7 & 24.0 & 84.1 & 54.0 & 98 & 40.8 \\
\hline June & 23.2 & 34.2 & 28.7 & 35.3 & 83.1 & 59.2 & 70 & 29.2 \\
\hline July & 25.3 & 34.5 & 29.9 & 45.9 & 84.5 & 65.2 & 54 & 22.5 \\
\hline Aug & 25.6 & 34.4 & 30.0 & 50.4 & 84.9 & 67.7 & 91 & 37.9 \\
\hline Sep. & 23.7 & 34.1 & 28.9 & 39.8 & 84.5 & 62.2 & 78 & 32.5 \\
\hline Oct. & 20.7 & 30.8 & 25.8 & 37.8 & 83.5 & 60.7 & 44 & 18.3 \\
\hline
\end{tabular}

Table 10. Relation between averages of monthly fluctuation abundance and temperature $\&$ relative humidity $(\mathrm{RH})$ of some rodent species in Sharkia Governorate during (Nov. 2005 - Oct. 2006).

\begin{tabular}{|c|c|c|c|c|c|c|c|c|}
\hline Months & $\begin{array}{c}\text { Min } \\
\text { temp }\end{array}$ & $\begin{array}{c}\text { Max } \\
\text { temp }\end{array}$ & $\begin{array}{c}\text { Mean } \\
\text { temp }\end{array}$ & $\begin{array}{c}\text { Min } \\
\text { RH\% }\end{array}$ & $\begin{array}{c}\text { Max } \\
\text { RH\% }\end{array}$ & $\begin{array}{c}\text { Mean } \\
\text { RH\% }\end{array}$ & $\begin{array}{c}\text { Total No. } \\
\text { of rats }\end{array}$ & $\%$ \\
\hline Nov.04 & 16.0 & 26.3 & 21.1 & 39.9 & 84.0 & 62.0 & 38 & 15.8 \\
\hline Dec. & 14.2 & 22.9 & 18.5 & 49.6 & 83.8 & 66.7 & 45 & 18.8 \\
\hline Jan.05 & 10.1 & 20.2 & 15.1 & 42.9 & 85.9 & 64.4 & 38 & 15.8 \\
\hline Feb. & 12.0 & 22.4 & 17.2 & 63.0 & 80.3 & 71.6 & 29 & 12.1 \\
\hline Mar. & 13.2 & 27.0 & 20.1 & 33.6 & 83.7 & 58.6 & 18 & 7.5 \\
\hline Apr. & 15.3 & 28.8 & 22.1 & 33.1 & 83.3 & 58.2 & 58 & 24.2 \\
\hline May & 19.5 & 31.8 & 25.6 & 25.2 & 84.1 & 54.7 & 73 & 30.4 \\
\hline June & 24.0 & 35.6 & 29.8 & 35.3 & 85.5 & 60.4 & 50 & 20.8 \\
\hline July & 26.1 & 34.9 & 30.5 & 47.1 & 85.1 & 66.1 & 40 & 16.7 \\
\hline Aug & 25.6 & 35.0 & 30.3 & 47.5 & 85.1 & 66.3 & 63 & 26.3 \\
\hline Sep. & 23.5 & 34.4 & 29.0 & 41.7 & 84.4 & 63.0 & 38 & 15.8 \\
\hline Oct. & 21.4 & 31.3 & 26.3 & 41.2 & 84.3 & 62.8 & 41 & 17.1 \\
\hline
\end{tabular}

Correlation between mean of temperature \& relative humidity $(\mathrm{RH})$ and the total number of rats during two years of the study.

\begin{tabular}{|c|c|c|}
\hline \multirow{2}{*}{} & \multicolumn{2}{|c|}{ Total number of rats } \\
\cline { 2 - 3 } & $2004 / 2005$ & $2005 / 2006$ \\
\hline Mean Temp & $0.652^{*}$ & 0.421 \\
\hline Mean RH\% & -0.265 & -0.380 \\
\hline
\end{tabular}




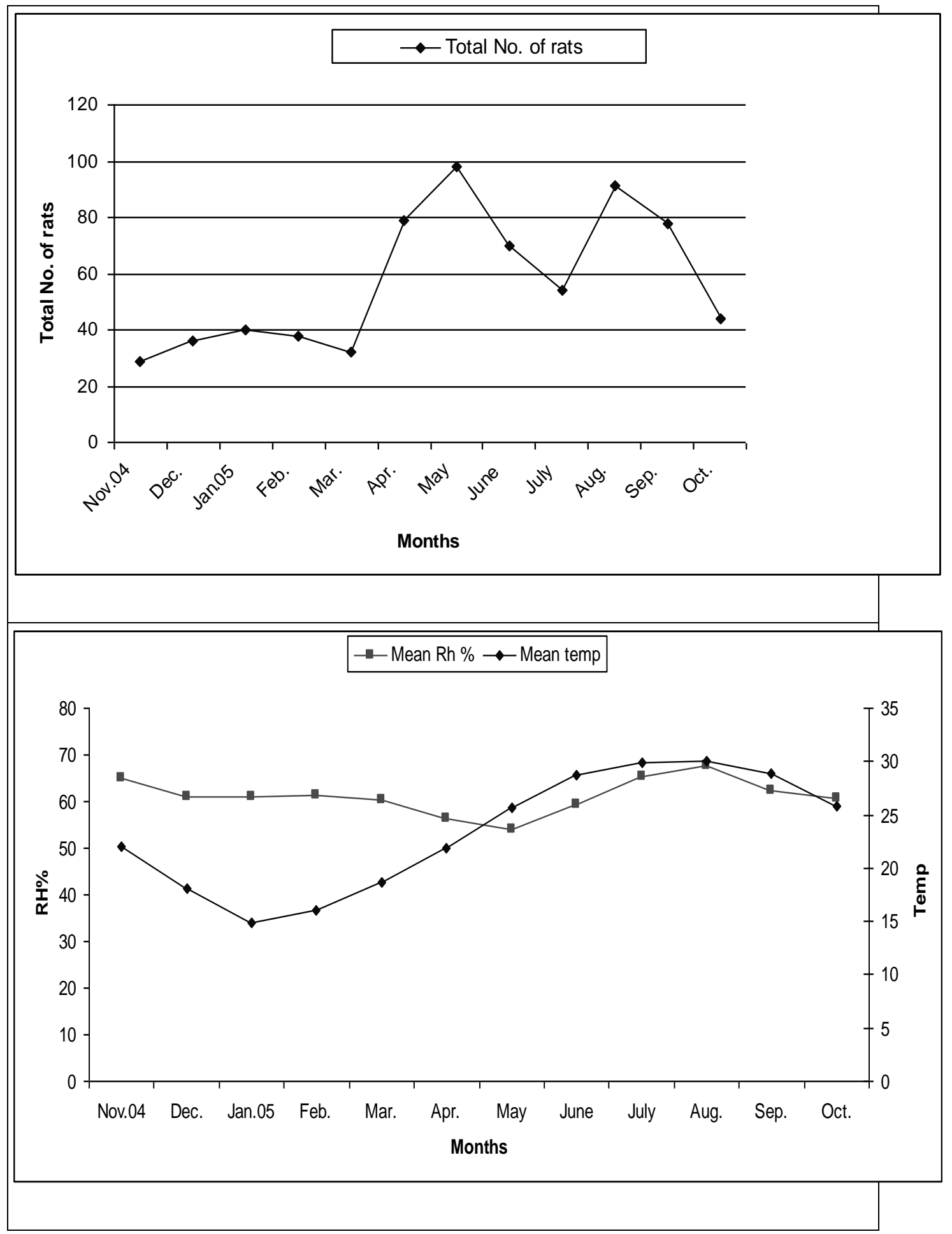

Fig 1. Relation between averages of monthly fluctuation abundance and temperature $\&$ relative humidity $(\mathrm{RH})$ of some rodent species in Sharkia Governorate during (Nov. 2004- Oct. 2005). 


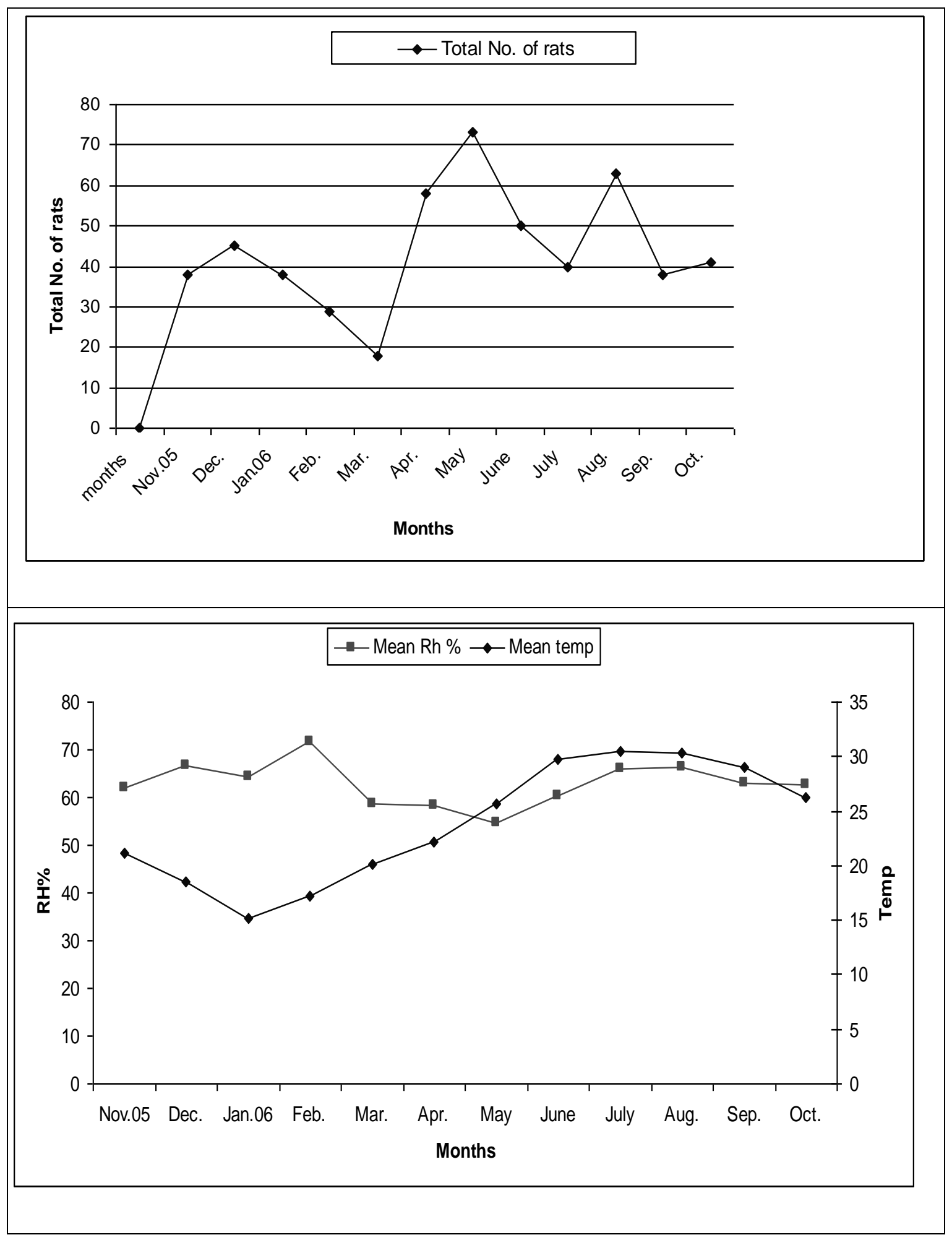

Fig 2. Relation between averages of monthly fluctuation abundance and temperature \& relative humidity $(\mathrm{RH})$ of some rodent species in Sharkia Governorate during (Nov. 2005 - Oct. 2006). 


\section{REFERENCES}

1. Abdel- El- Azeem, M.I. 2008. Ecological studies on some commensal rodent species and their ectoparasites in different habitats at Sharkia governorate. M. Sc. Thesis, Fac. Agric. Suez Canal. University. pp 193.

2. Abdel- El- Karim, S.M. 1991. Studies on rodents in Sharkia Governorate. Ph. D. Thesis, Fac. of Agric. Zagazig Univ. Egypt.

3. Abdel - Gawad, K.H. 1974. Ecological and toxicological studies on commensal and household rodents in Assiut area. M. Sc. Thesis, Fac. Of Agric., Assiut Univ., Egypt.

4. Abdel- Gawad, K.H. 1979. Studies on the interrelation. Between rodents and their ectoparasites in the cultivated and semiarid. Zones. Ph. D. Thesis, Fac. Of Agric., Assiut Univ., Egypt.

5. Abdel - Gawad, K.H., A.M. Salit and A. Maher- Ali. 1982. Population density of rodent species in agricultural and semi-arid areas. Assuit J. Agric. Sci., 13 (2): 27 $-37$.

6. Asran, A.A. 1994. Population dynamics and reproduction aspects of the Nile rat Arvicanthis niloticus. Egypt. J. Agric. Res., 72 (2): 427 - 432.

7. Asran, A.A., H.I. El-Deeb, G. Kuehrnert and M.A. El-Halafawy. 1985. Population density of rodent in different locations in Fayoum Governorate. J. Agric. Sci. Mansoura Univ. Egypt, 10 (4): 1527 - 1528.

8. Asran, A.A., H.I. El-Deeb and M.A. El-Halafawy. 1991. Rat damage to certain crops and population density of $A$. nilaticas in Fayoum Governorate. Egypt. J. Agric. Res., 69 (1): $273-280$.

9. Bajomi, D. 1984. Commensal rodent proplems in Hungry Organization and practice of vertebrate pest control. Hampshire Conference, England: $18-20$.

10. Dykstra, W.W. 1966. The economic importance of commensal rodents. WHO / Vector Control, $66: 217$.

11. El-Bahrawy, A.A. F. 1986. Studie on ecology and control of some rodent species in Ismailia Governorate -Ph.D. Thesis, Fac. Of Agric. Suze canal, Univ., Egypt.

12. El-Bahrawy, A.A.F., A.M.I. Hegab, G.A. El-Kady and M.I.A. El-bkhshwangi 2008. Field studies on commensal rodents at El-Ibrahemia District, Sharkia Governorate. Egypt J. Appl. Sci., 23 (5): 230 - 243. 
13. Embarak, M.Z. 1997. Ecological and control studies on rodents and their ectoparasites in cultivated and newly reclaimed areas. M. Sc. Thesis, Fac. Agric. Assiut Univ.

14. Gaaboub, I. A., A. E.E. Widaatalla and M.F. El-Sawy. 1978. Seasonal of small rodents in relation to cultivated area in the vicinity of Alexandria Governorate, Egypt. J. Agric. Res. Tanta, Univ., 4 (2): 312 - 322.

15. Hegab, A.M.I. 2004. Studies on some commensal rodent species and their ectoparasites in different habitats at Sharkia Governorate. Agric. Res. J. Suez Canal Univ., 4 (2): $119-124$.

16. Hegab, A.M.I., Sh.A.A. Ismail and S.A.A. El-Massry. 2006. Survey, morphological studies and ectoparasites associated with some commansal rat species at Sharkia Governorate. Egypt. J. Appl. Sci., 21 (5): 340- 349.

17. Mourad, M.G., K.H. Abdel -Gawad and A. Maher Ali. 1982. Population density of rodent species in Some Urban area in Minia Governorate. Assiut J. Agric. Sci., Egypt., 13 (2): $19-25$.

18. Mmetwaly, A.M., S. A. Montasser and A. A. R. Al-Gendy. 2009. Survey of Rodent Species and Damage Assessment Caused by Meriones shawi isis (Thomas) in Some Field Crops at Bustan Area . J. Appl. Sci. Res., 5(1): 40-45.

19. Omar, M.T. 1980. A Preliminary survey and seasonal population on domestic and commensal rodents in $10^{\text {th }}$ of Ramadan city as a non -inhabited area. Proc. Conf. PI. Protec. Res. In., 3: 269 - 274. Int. Conference Plant Protection Res

20. Osbron, D.J. and I. Helmy. 1980. The contemporary land mammals of Egypt (including Sinai). Fieldiana Zoology new series 5: 1-579

21. Soluman, S., A.J. Main, A.S. Marzouk and A.A. Montasser. 2001. Seasonal studies on commensal rats and their ectoparasites in rural area of Egypt: the relationship of ectoparasites to the species, locality, and relative abundance of the host. J. Parasitol., 87 (3): 545 - 553. 


\section{حصر وتذبذب تعداد بعض أنواع الجرذان فى ثلاث مواطن مختلفة بمركز الإبر اهيمية محافظة الثرقية}

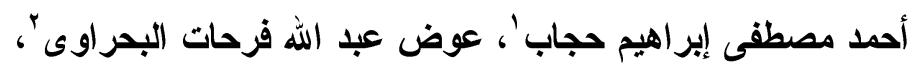

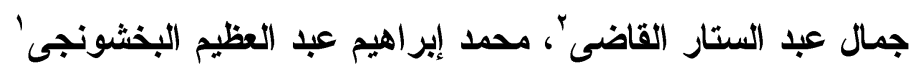

$$
\text { قعهم وقاية النباتات - كلبة الزراعة النباتات - مركز البحوث الزراعبة- الدقي جيزة' }
$$

الهدف من هذا البحث هو حصر بعض أنواع القوارض فيى ثلاث مو اقــع مختلفـــة بمحافظــــة

$$
\begin{aligned}
& \text { الثرقية وأظهرت النتائج أن الجرزان المصاده تتقسم إلى الآتى: }
\end{aligned}
$$

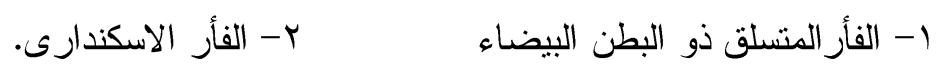

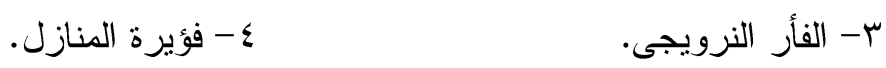

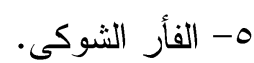

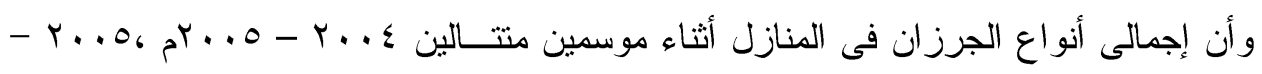

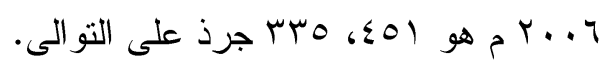

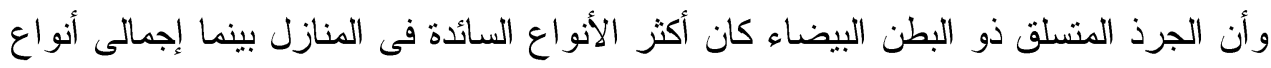

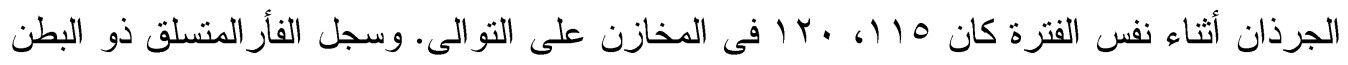

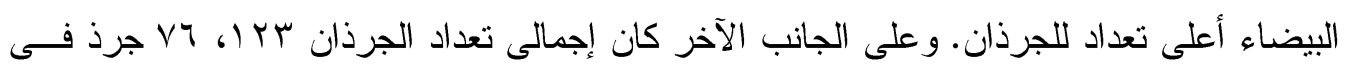

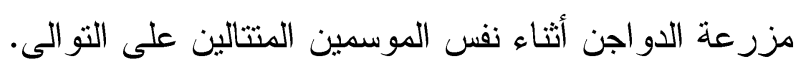

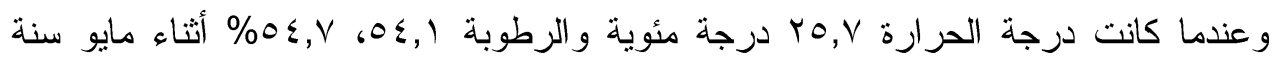

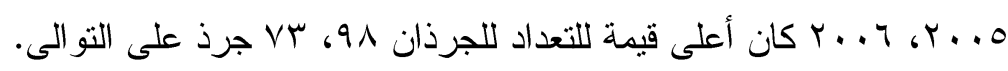

